\title{
Levaduras aisladas de mieles como antagonistas de mohos patógenos
}

\section{de cultivos}

\author{
Alfonso Emanuel Carrizo Villoldo ${ }^{1,2, *}$; Carla Belén Carrizo ${ }^{3}$; Marcelo Rafael Benítez \\ Ahrendts ${ }^{4}$; Leonor Carrillo 4 \\ ${ }^{1}$ Cátedra de Microbiología General, Facultad de Ciencias Agrarias, UNJu, Jujuy, Argentina, C.P. 4600; \\ ${ }^{2}$ CONICET; ${ }^{3}$ INTA-IPAF Región NOA, Ruta Nacional Nº 9 km 1763, Posta de Hornillos, Departamento \\ Maimará, Jujuy, Argentina, C.P. 4622; ${ }^{4}$ Cátedra de Microbiología Agrícola, Facultad de Ciencias Agrarias, \\ UNJu, Jujuy, Argentina, C.P. 4600; *alfonsocarrizo@fca.unju.edu.ar
}

Carrizo Villoldo, Alfonso Emanuel; Carla Belén Carrizo; Marcelo Rafael Benítez Ahrendts; Leonor Carrillo (2020). Levaduras aisladas de mieles como antagonistas de mohos patógenos de cultivos. Rev. Fac. Agron. Vol 119 (2): 1-11. https://doi.org/10.24215/16699513e054

\begin{abstract}
Se buscó el aislamiento de levaduras de muestras de miel de la Provincia de Jujuy, Argentina, con el objetivo de utilizarlas in vitro como antagonistas de mohos patógenos pre- y poscosecha, de diferentes cultivos de interés, como, cítricos, maíz, maní y caña de azúcar. Se utilizó miel, ya que la misma puede actuar como medio selectivo de cepas inocuas y tolerantes a distintos estrés abióticos, como bajo pH, elevada presión osmótica, presencia de fitoquímicos, entre otros. Se logró aislar un total de 15 levaduras de 25 muestras de mieles. Las levaduras fueron identificadas como: Candida parapsilosis, Zygosaccharomyces baili, Zygosaccharomyces mellis, Zygosaccharomyces rouxii, Tausonia pullulans, Lachancea thermotolerans, Lachancea fermentati, Torulaspora delbruecki y Saccharomyces cerevisiae. Todos los géneros se encontraron descriptos como presentes en la miel, provenientes, ya sea, de fuentes primarias de contaminación o del ambiente circundante. De los aislamientos obtenidos se probó el antagonismo in vitro, por disminución del crecimiento micelial de los mohos: Penicillium italicum, P. ulaiense, Aspergillus parasiticus y Fusarium sacchari. Lachancea thermotolerans, fue el único aislamiento que presentó antagonismo hacia todos los mohos ensayados. Candida parapsilosis manifestó antagonismo hacia $P$. italicum; Lachancea fermentati hacia $P$. ulaiense; $Z$. mellis frente $P$. ulaiense y uno de los aislamientos de $T$. delbrueckii frente a $P$. italicum y $P$. ulaiense. Se podrían utilizar las levaduras antagonistas en futuros ensayos in vivo, en vistas al diseño de un biofungicida activo contra mohos patógenos de cultivos, en la etapa de producción a campo o durante el almacenamiento.
\end{abstract}

Palabras clave: levaduras; mohos; patógenos; precosecha; poscosecha

Carrizo Villoldo, Alfonso Emanuel; Carla Belén Carrizo; Marcelo Rafael Benítez Ahrendts; Leonor Carrillo (2020). Yeasts isolated from honeys as antagonists of crop pathogenic molds. Rev. Fac. Agron. Vol 119 (2): 1-11. https://doi.org/10.24215/16699513e054

The isolation of yeasts from honey samples from the Province of Jujuy, Argentina, was sought with the aim of using them in vitro as antagonists of pre-and postharvest pathogenic molds of different crops of interest, such as, citrus fruits, peanut and sugar cane. Honey was used, since it can act as a selective medium for innocuous strains, and tolerant to different abiotic stresses, such as low $\mathrm{pH}$, high osmotic pressure, presence of phytochemicals, among others. A total of 15 yeasts were isolated from 25 honey samples. Yeasts were identified as: Candida parapsilosis, Zygosaccharomyces baili, Zygosaccharomyces mellis, Zygosaccharomyces rouxii, Tausonia pullulans, Lachancea thermotolerans, Lachancea fermentati, Torulaspora delbrueckii y Saccharomyces cerevisiae. All genera were founded to be described as present in honey, originated from either primary sources of contamination, or the surrounding environment. Of the isolates obtained, antagonism was tested in vitro, by decreasing the mycelial growth of molds: Penicillium italicum, P. ulaiense, Aspergillus parasiticus and Fusarium sacchari. Lachancea thermotolerans, was the only isolation that presented antagonism towards all the molds tested. Candida parapsilosis presented antagonism towards $P$. italicum, Lachancea fermentati towards $P$. ulaiense, $Z$. mellis against $P$. ulaiense, and one of the isolates of $T$. delbrueckii against $P$. italicum and $P$. ulaiense. Antagonistic yeasts could be used, in future in vivo tests, in view of the design of an active biofungicide against crop pathogenic molds, at field production stage, or during storage.

Key words: yeasts; molds; pathogenic; preharvest; postharvest

https://revistas.unlp.edu.ar/revagro

Recibido: 22/08/2019

Aceptado: 02/10/2019

Disponible on line: $07 / 12 / 2020$

ISSN 0041-8676 - ISSN (on line) 1669-9513, Facultad de Ciencias Agrarias y Forestales, UNLP, Argentina. 


\section{INTRODUCCIÓN}

Las especies fúngicas son agentes etiológicos de un gran número de enfermedades pre- y postcosecha de cultivos (Chen et al., 2007). Muchas especies del género Fusarium son fitopatógenas causando enfermedades como marchitamiento vascular, necrosis de raíces y tallos y, además, producen durante el almacenamiento de granos, micotoxinas como la beauvericina y fumonisina, que son perjudiciales para la salud animal (Leslie \& Summerell, 2006; Moretti et al., 2007; Döll \& Dänicke, 2011; Viswanathan et al., 2011). Entre los cultivos afectados se encuentran maíz y caña de azúcar (Ploetz, 2006; Viswanathan et al., 2011).

Otro género productor de micotoxinas es Aspergillus, cuyas especies $A$. flavus y $A$. parasiticus son las principales productoras de aflotoxinas, que son carcinogénicas para humanos y animales (Williams et al., 2004; Pitt \& Hocking, 2009) y pueden estar presentes en diferentes etapas de la cadena de comercialización del maní (Martins et al., 2017).

En cuanto a las pérdidas postcosecha de frutas por mohos, se han reportado pérdidas de entre el $25 \%$ y $50 \%$ (Nunes, 2012). Entre las principales enfermedades que afectan a este grupo de frutas son mohos del género Penicillium (Pitt \& Hocking, 2009). Estos hongos son patógenos de heridas y causan una podredumbre que deteriora rápidamente las características organolépticas de la fruta (Nunes, 2012).

Para controlar estas enfermedades, el uso de fungicidas sintéticos es el método más común, debido a su costo relativamente bajo, facilidad de aplicación y efectividad. Sin embargo, su efectividad se ve limitada debido a la alta frecuencia de aparición de cepas resistentes (Chen et al., 2007; Sánchez-Torres \& Tuset, 2011). Además, su uso es cada vez más restringido debido a su alta toxicidad residual, efectos carcinogénicos, degradación y contaminación ambiental generada (Tripathi \& Dubey, 2003; Palou et al., 2008).

En los últimos 20 años, las levaduras han ganado potencial como agentes de control biológico de mohos, debido a su rápida tasa de crecimiento, no producen esporas alergénicas o micotoxinas y poseen necesidades nutricionales simples (Hua et al., 1999; Nally et al., 2012; Guo et al., 2014). Por este motivo, es factible utilizar levaduras antagónicas para combatir mohos patógenos en frutas y verduras, como alternativa para reducir, o incluso reemplazar, a los fungicidas sintéticos (Janisiewicz et al., 2000; Sharma et al., 2009; Droby et al., 2009).

Asimismo, varios mecanismos de acción están involucrados en los procesos de control biológico de patógenos fúngicos. Los mismos, se basan generalmente en la capacidad de adherirse a sitios específicos, colonizar heridas y competir por nutrientes (Liu et al., 2013a); secretar enzimas específicas como glucanasas y quitinasas con acción lítica en la pared celular de hongos (Castoria et al., 2001; Hu et al., 2017); inducir resistencia en la planta (Yao \& Tian, 2005); inhibición del crecimiento micelial de mohos por metabolitos difusibles y volátiles (Huang et al., 2011; Lutz et al., 2013); formar un biofilm en la superficie interna de heridas (Giobbe et al., 2007) y la producción de toxinas 'killer', letales para otras levaduras susceptibles (Marquina et al., 2002), así como para las hongos filamentosos y bacterias (Platania et al., 2012; Bajaj et al., 2012; Al-Qaysi et al., 2017).

El uso de la miel para el tratamiento de enfermedades data de unos 2000-2100 años a. C.; incluso Aristóteles (384-322 a. C.) la describió como buena para el tratamiento de heridas (Mandal \& Mandal, 2011; Vallianou et al., 2014).

Las propiedades antimicrobianas, se le han atribuido principalmente al peróxido de hidrógeno y a varios compuestos fitoquímicos, como flavonoides, fenoles y ácidos orgánicos; por este motivo tiene un número reducido de microorganismos, y sólo se encuentran viables, aquellos que están adaptados a estas condiciones (Snowdon \& Cliver, 1996; Bakier, 2009; Gomes et al., 2011; Velásquez Giraldo et al., 2013).

Además, la alta osmolaridad y baja actividad agua no permiten el desarrollo de microorganismos que provienen de fuentes de difícil control, como el polvo, el aire, el suelo y el néctar (Snowdon \& Cliver, 1996; Spencer \& Spencer, 1997; Vázquez-Quiñones et al., 2018).

Los microorganismos presentes en la miel pueden influenciar su estabilidad y calidad higiénica (Vica et al., 2009). Entre los microorganismos presentes en la miel, han sido reportados, bacterias, levaduras y mohos; y géneros como Penicillium, Mucor, Saccharomyces, Schizosaccharomyces, Zygosaccharomyces y Torulaspora, son responsables de su fermentación, cuando el contenido de humedad supera el 19-20\% (Snowdon \& Cliver, 1996; Codex Alimentarius, 2001). Fusarium y Aspergillus también fueron descriptos como frecuentes en muestras de miel (Vica et al., 2009).

Si bien, la mayor parte de las levaduras en la miel no pueden crecer o se encuentran como viables pero no cultivables (VPNC), debido principalmente a la alta concentración de azúcar y antimicrobianos (Déak, 2008); se detectó la producción de etanol en algunas muestras de miel almacenada; indicando que hay ciertos tipos de levaduras, que pueden haber sufrido una domesticación natural, y convertir los carbohidratos en etanol, aún bajo alto estrés de azúcar (Liu et al., 2016); y otros tipos de estrés, como, fitoquímicos provenientes del néctar (Herrera et al., 2009; Herrera et al., 2010).

En el presente trabajo se buscó aislar distintas especies de levaduras, en lo posible inocuas, y tolerantes a diferentes estrés inherentes a la miel, por su naturaleza antimicrobiana; y que puedan presentar antagonismo hacia mohos patógenos pre- y poscosecha de cultivos.

\section{MATERIALES Y MÉTODOS}

\section{Muestras}

El muestreo se efectuó en apiarios pertenecientes a productores de la zona este de la Provincia de Jujuy, en la zona de valles, yungas y chaco, durante la época estival de 2008-2009. La toma de muestras de miel se realizó en panales operculados (miel madura) y sin opercular (miel inmadura) de Apis mellifera L. Se tomaron aproximadamente $200 \mathrm{~g}$ en envases estériles. Para controlar la medida de la acidez o alcalinidad de las muestras de miel se utilizó un medidor de $\mathrm{pH}$ Testo 206-pH2. 
Los sólidos solubles totales de las muestras de miel ( ${ }^{\circ}$ Brix) fueron medidos a $20^{\circ} \mathrm{C}$ utilizando un refractómetro de mano Westover RHB-18, las muestras se diluyeron cuatro veces con agua destilada. A partir de los ${ }^{\circ}$ Brix obtenidos se calculó el porcentaje de humedad $(\% \mathrm{H})$ según tablas de Chataway (Canadian Food Inspection Agency, 2019)

\section{Aislamiento de levaduras}

Se llevaron a cabo en el laboratorio de la Cátedra de Microbiología Agrícola, de la Facultad de Ciencias Agrarias (FCA), Universidad Nacional de Jujuy (UNJu). Se utilizó miel recién recolectada o almacenada por un periodo no mayor a 3 meses. Se efectuó por la técnica de estrías en placas de Petri, utilizando los medios agar extracto de malta (MEA g/l: extracto de malta 20; peptona 1; glucosa 20; agar 20), agar diclorán rosa bengala cloranfenicol (DRBC g/L: glucosa 10; peptona 5; fosfato dihidrógeno de potasio 1; sulfato de magnesio 0,5 ; solución de rosa bengala al $5 \% \mathrm{p} / \mathrm{v} \quad 0,5 \mathrm{ml}$; cloranfenicol 0,1 ; diclorán $0,2 \% \mathrm{p} / \mathrm{v}$ en etanol $1 \mathrm{ml}$; agar 15), agar diclorán 18\% glicerol (DG18 g/L: glucosa 10; peptona 5; fosfato dihidrógeno de potasio 5 ; sulfato de magnesio 0,5 ; cloranfenicol 0,1 ; diclorán $0,2 \% \mathrm{p} / \mathrm{v}$ en etanol $1 \mathrm{ml}$; glicerol 220; agar 15), agar czapek glicerol (G25N g/L: fosfato monohidrógeno de potasio 0,75 ; solución de czapek 7,5 ml; extracto de levadura 3,75; glicerol 250; agar 15) (Pitt \& Hocking, 2009). Las levaduras aisladas, fueron mantenidas a $4^{\circ} \mathrm{C}$ en MEA.

\section{Identificación de las levaduras}

La identificación se realizó a través de pruebas fisiológicas siguiendo la metodología de Déak (2008) con modificaciones. Se utilizó medio base con nitrógeno ( $\mathrm{g} / \mathrm{L}$ : sulfato de amonio 5 , fosfato dihidrógeno de potasio 5 , sulfato de magnesio 0,5 ) al que se le agregó los diferentes compuestos carbonados en una concentración de 0,02 $\mathrm{g} / \mathrm{ml}$ para observar la asimilación de azúcares o polialcoholes (glucosa, galactosa, celobiosa, lactosa, maltosa, melibiosa, trehalosa, melezitosa, rafinosa, arabinosa, ramnosa, xilosa, mesoeritritol, myo-inositol, manitol).

Para la prueba de asimilación de fuentes de nitrógeno, se utilizó medio base con carbono ( $\mathrm{g} / \mathrm{L}$ : glucosa 10; fosfato dihidrógeno de potasio 1; sulfato de magnesio 0,5). Luego se agregó como fuentes nitrogenadas: nitrato de sodio y cadaverina, en una concentración de $0,001 \mathrm{~g} / \mathrm{ml}$.

También se realizaron pruebas de fermentación de azúcares (glucosa y sacarosa), hidrólisis de la urea, crecimiento en medio con $0,01 \%$ de cicloheximida y asimilación de lisina. Asimismo, se evaluó el crecimiento en medios con diferente concentración de azúcar, sal y ácidos orgánicos según Pitt \& Hocking (2009).

Se observó el crecimiento macroscópico y microscópico de las colonias en medio de cultivo sólido MEA, medio glucosa-peptona-extracto de levadura (GPY g/l: peptona 10; extracto de levadura 10; glucosa 40) y agar papa-dextrosa (g/L: glucosa 20; agar 20; infusión de papa $230 \mathrm{ml}$ : agua destilada $770 \mathrm{ml}$ ) a los 3 y 7 días a 25-28 ${ }^{\circ} \mathrm{C}$. Se tuvieron en cuenta características macroscópicas como: superficie, forma, brillo, consistencia, color, si producen cambio de color en el medio, elevación, margen, diámetro, producción de película. En cuanto a las características microscópicas, se observó: forma, gemación, presencia de pseudomicelio, tamaño; mediante un microscopio Zeiss modelo 473011/9901. Las muestras microscópicas se tiñeron, con azul de algodón o lactofenol.

También se evaluó esporulación por un lapso de 6 semanas utilizando agar Mc Clary (g/L: glucosa 1; cloruro de potasio 1,8; acetato de potasio 8,2; extracto de levadura 2,5; agar 15); los cultivos fueron observados microscópicamente con contraste de fases o por tinción de Wirtz-Conklin.

Los resultados obtenidos con las claves taxonómicas de Déak (2008), fueron comparados con claves y fotografías de Kurtzman et al. (2011) y la Polyphasic Identification del Westerdijk Fungal Biodiversity Institute (2019). Los nombres científicos fueron actualizados según la base de datos del Mycobank Database (Robert et al., 2004), último acceso en mayo de 2019.

\section{Acción inhibitoria in vitro de levaduras sobre mohos}

Se probó el efecto antagónico de levaduras obtenidas de mieles frente a mohos patógenos poscosecha de cítricos: Penicillium italicum Wehmer y $P$. ulaiense H.M. Hsieh, H.J. Su \& Tzean; y otros patógenos de maíz, maní y caña de azúcar: Aspergillus parasiticus Speare y Fusarium sacchari (E.J. Butler) W. Gams. Todos pertenecientes al cepario de la Cátedra de Microbiología Agrícola (FCA-UNJu).

Se utilizó la técnica de Hua et al. (1999) con modificaciones, se sembraron las cepas de mohos en MEA e incubaron a $25-28^{\circ} \mathrm{C}$ durante 7 días. Luego se tomaron conidios del centro de una colonia mediante un asa y se colocaron en $5 \mathrm{ml}$ de agar al 0,2\%. La suspensión de levaduras se preparó con aislados mantenidos en MEA durante $48 \mathrm{~h}$ a $25-28^{\circ} \mathrm{C}$. Se llevó a una concentración de $10^{5}$ células $/ \mathrm{ml}$ en agua estéril, por recuento en cámara de Neubauer.

Luego se sembró la solución de conidios mediante punciones cada $1 \mathrm{~cm}$ a lo largo de una estría de $6 \mathrm{~cm}$ de longitud en el centro de una placa de Petri de $9 \mathrm{~cm}$ de diámetro con MEA. Se incubó a $25^{\circ} \mathrm{C}$ durante $24 \mathrm{~h}$. Pasadas las $24 \mathrm{~h}$ se sembró $10 \mu \mathrm{L}$ de la suspensión de levaduras en dos estrías de $6 \mathrm{~cm}$ ubicadas a ambos lados y a $1,5 \mathrm{~cm}$ de la estría central que contenía el moho.

Se realizó una siembra testigo, donde se sembró al moho correspondiente, pero no a las levaduras. A los 7 días se registró el espesor del crecimiento del moho en milímetros, y se comparó con el testigo. Las pruebas se realizaron por duplicado.

\section{Análisis estadístico}

Con los datos de espesor en milímetros que registraron los mohos, se realizó un análisis de varianza de una vía (ANOVA), seguido de la prueba de comparaciones múltiples de Dunnett, mediante el paquete estadístico Minitab versión 18.1. Las diferencias fueron aceptadas como significativas cuando $p<0,05$.

\section{RESULTADOS}

Se recolectaron un total de 24 muestras de mieles de Apis mellifera de apiarios de diferentes zonas 
productivas de la Provincia de Jujuy, una de las muestras correspondió a Tetragonisca angustula (Latreille) (abeja melipona) proveniente de Orán, Provincia de Salta. Las muestras presentaron una medida del $\mathrm{pH}$ de entre 3,4 hasta 5,3 y una humedad entre $17,5 \%$ hasta menos de $13,0 \%$; aunque los aislamientos de levaduras fueron obtenidos de muestras de entre el 13,0 y $16,3 \%$ de humedad (Tabla 1).

\begin{tabular}{|c|c|c|c|c|c|c|c|c|}
\hline Muestra & Origen & $\begin{array}{c}\text { Tipo de } \\
\text { miel }\end{array}$ & $\mathrm{pH}$ & ${ }^{\circ}$ Brix & $\% \mathrm{H}$ & $\begin{array}{c}\text { Medio de } \\
\text { aislamiento }\end{array}$ & Aislamiento/s & Especie/s \\
\hline 1 & Los Alisos $^{a}$ & Madura & 3,6 & 80,9 & 17,5 & - & - & - \\
\hline 2 & Los Alisos ${ }^{a}$ & Madura & 3,4 & 81,0 & 17,4 & - & - & - \\
\hline 3 & S. S. de Jujuy a & Madura & 3,9 & 82,4 & 16,1 & G25N & $5 ; 7$ & $\begin{array}{l}\text { Torulaspora } \\
\text { delbrueckii** }\end{array}$ \\
\hline 4 & La Almona ${ }^{a}$ & Madura & 3,7 & 84,8 & 13,8 & DG18 & 6 & $\begin{array}{l}\text { Tausonia } \\
\text { pullulans }\end{array}$ \\
\hline 5 & S. S. de Jujuy a & Madura & 3,6 & 82.3 & 16,2 & - & - & - \\
\hline 6 & Bárcena b & Madura & 3,8 & 89,3 & $13,0^{*}$ & MEA & 9 & $\begin{array}{c}\text { Torulaspora } \\
\text { delbrueckii }\end{array}$ \\
\hline 7 & Bárcena ${ }^{b}$ & Inmadura & 4,1 & 89,8 & $13,0^{*}$ & MEA & 4 & $\begin{array}{c}\text { Lachancea } \\
\text { fermentati } \\
\text { Lachancea }\end{array}$ \\
\hline 8 & Finca Escaro a & Madura & 5,3 & 86,2 & $13,0^{*}$ & MEA & $3 ; 10$ & $\begin{array}{l}\text { thermotolerans; } \\
\text { Zygosaccharom } \\
\text { yces bailii }\end{array}$ \\
\hline 9 & Finca Escaro a & Inmadura & 4,7 & 86,0 & $13,0^{*}$ & MEA & $2 ; 11$ & $\begin{array}{c}\text { Zygosaccharom } \\
\text { yces bailii; } \\
\text { Zygosaccharom } \\
\text { yces rouxii }\end{array}$ \\
\hline 10 & Los Alisos ${ }^{a}$ & Inmadura & 4,0 & 82,2 & 16,3 & MEA & 1 & $\begin{array}{c}\text { Candida } \\
\text { parapsilosis }\end{array}$ \\
\hline 11 & Los Alisos ${ }^{a}$ & Madura & 4,2 & 83,3 & 15,2 & - & - & - \\
\hline 12 & El Ceibal a & Madura & 3,9 & 85,6 & 13,0 & - & - & - \\
\hline 13 & Lobatón ${ }^{c}$ & Madura & 3,5 & 86,4 & $13,0^{*}$ & - & - & - \\
\hline 14 & $\begin{array}{l}\text { Los } \\
\text { Blancos }{ }^{a}\end{array}$ & Madura & 4,9 & 85,6 & 13,0 & - & - & - \\
\hline 15 & El Remate ${ }^{d}$ & Madura & 4,5 & 82,3 & 16,2 & - & - & - \\
\hline 16 & Palpalá d & Madura & 4,4 & 82,0 & 16,5 & - & - & - \\
\hline 17 & Palpalá d & Madura & 4,5 & 83,3 & 15,2 & - & - & - \\
\hline 18 & Sauce Huacho ${ }^{c}$ & Madura & 3,5 & 84,7 & 13,9 & MEA & 13 & $\begin{array}{c}\text { Zygosaccharom } \\
\text { yces mellis }\end{array}$ \\
\hline 19 & El Fuerte ${ }^{e}$ & Madura & 3,9 & 83,3 & 15,2 & - & - & - \\
\hline 20 & El Fuerte ${ }^{e}$ & Madura & 4,3 & 86,1 & $13,0^{*}$ & - & - & - \\
\hline 21 & El Fuerte ${ }^{e}$ & Madura & 4,0 & 84,6 & 14,0 & MEA & 12 & $\begin{array}{c}\text { Saccharomyces } \\
\text { cerevisiae }\end{array}$ \\
\hline 22 & El Fuerte ${ }^{e}$ & Madura & 4,1 & 84,4 & 14,2 & MEA & 14 & $\begin{array}{c}\text { Torulaspora } \\
\text { delbrueckii }\end{array}$ \\
\hline 23 & El Fuerte e & Madura & 4,3 & 84,2 & 14,4 & - & - & - \\
\hline 24 & El Fuerte ${ }^{e}$ & Madura & 4,3 & 85,4 & 13,2 & MEA & 15 & $\begin{array}{l}\text { Torulaspora } \\
\text { delbrueckii }\end{array}$ \\
\hline 25 & Orán ${ }^{f}$ & Madura & - & - & - & MEA & 8 & $\begin{array}{c}\text { Zygosaccharom } \\
\text { yces mellis }\end{array}$ \\
\hline
\end{tabular}

Tabla 1. Origen, tipo de miel (madura o inmadura), características fisicoquímicas ( $\mathrm{pH}$, ${ }^{\circ} \mathrm{Brix}$ y \%H), medio de aislamiento (DG18, G25N ó MEA), aislamiento/s (numerados del 1 al 15) y especie/s de levaduras identificadas de la totalidad de muestras de miel. a Departamento Dr. Manuel Belgrano; $b$ Departamento Tumbaya; ' Departamento Santa Bárbara; ${ }^{d}$ Departamento Palpalá; e Departamento San Pedro; ${ }^{\prime}$ Departamento Orán, Provincia de Salta; * contenido de humedad menor al 13\%; ** ambos aislamientos fueron de la misma especie. 
De las 25 muestras de miel, se aisló un total de 15 levaduras, siendo el agar MEA el más utilizado. También se utilizó agar $\mathrm{G} 25 \mathrm{~N}$ en el caso de las levaduras 5 y 6 , y agar DG18 sólo en el caso de la levadura 6 (Tabla 1).

La levadura $N^{\circ} 6$, obtenido de la muestra $N^{\circ} 4$, fue identificada como Tausonia pullulans (Lindner) X.Z. Liu, F.Y. Bai, M. Groenewald \& Boekhout. La misma, resultó positiva para la prueba de la ureasa, manifestando ser una levadura basidiomicética; el resto de los aislamientos dieron resultado negativo a la misma prueba. Además, presentó como características principales células con gemación, coloración beige, pseudomicelio con endosporas, fermentación ausente, myo-inositol positivo, nitrato positivo.

Las levaduras $\mathrm{N}^{\circ} 1,2,3,5,7,8,9,10,11,13,14$ y 15 , no asimilaron nitrato y eritritol, no crecieron en presencia de cicloheximida, pero si asimilaron manitol. Las mismas fueron identificadas como (Tabla 1): Levadura $\mathrm{N}^{\circ} 1$ : Candida parapsilosis (Ashford) Langeron \& Talice; Levadura N² y 10: Zygosaccharomyces bailii (Lindner) Guilliermond; Levadura $\mathrm{N}^{\circ} 3$ : Lachancea thermotolerans (Filippov); Levadura $\mathrm{N}^{\circ} 5,7$, 9, 14 y 15: Torulaspora delbrueckii (Lindner) Lindner; Levadura N`8 y 13: Zygosaccharomyces mellis Fabian \& Quinet; Levadura N¹1: Zygosaccharomyces rouxii (Boutroux) Yarrow.
La diferenciación entre $Z$. mellis y $Z$. rouxii, si bien se dificulta utilizando técnicas estándares de crecimiento, $Z$. rouxii es más tolerante a cloruro de sodio, permitiendo, así, su separación en un medio que contiene $16 \%$ de $\mathrm{NaCl}$ con $5 \%$ de glucosa. $Z$. baillii se diferenció de ambas al presentar crecimiento en agar con ácido acético al 1\%. T. delbrueckii, no se desarrolló en presencia de cadaverina, por lo que sirvió de criterio para diferenciarla de las especies nombradas anteriormente.

La levadura $N^{\circ} 4$, creció en presencia de $0,01 \%$ de cicloheximida, es celobiosa positiva, e incapaz de utilizar la lactosa, por lo que fue identificada como Lachancea fermentati $(\mathrm{H}$. Naganishi) Kurtzman.

La levadura $\mathrm{N}^{\circ} 12$, fue identificada como Saccharomyces cerevisiae Meyen ex E.C. Hansen; al presentar en medio GPY a los 7 días, células globosas, ovoidales o elongadas, y, principalmente, la ausencia de asimilación de manitol y lisina; asimilación positiva de maltosa, y crecimiento a $37^{\circ} \mathrm{C}$.

Se registró el diámetro de crecimiento en milímetros a los siete días de los mohos, sin la presencia de levaduras (figura $2 a, d, g, j$ ), y versus las levaduras $N^{\circ} 1$, $2,3,4,5,6,7,8$ y 9 , elegidas al azar. La levadura $N^{\circ} 3$ identificada como $L$. thermotolerans, se probó contra todas las cepas de mohos y manifestó antagonismo en todos los casos (Figura 1a-d y Figura 2b, 2e, 2h).
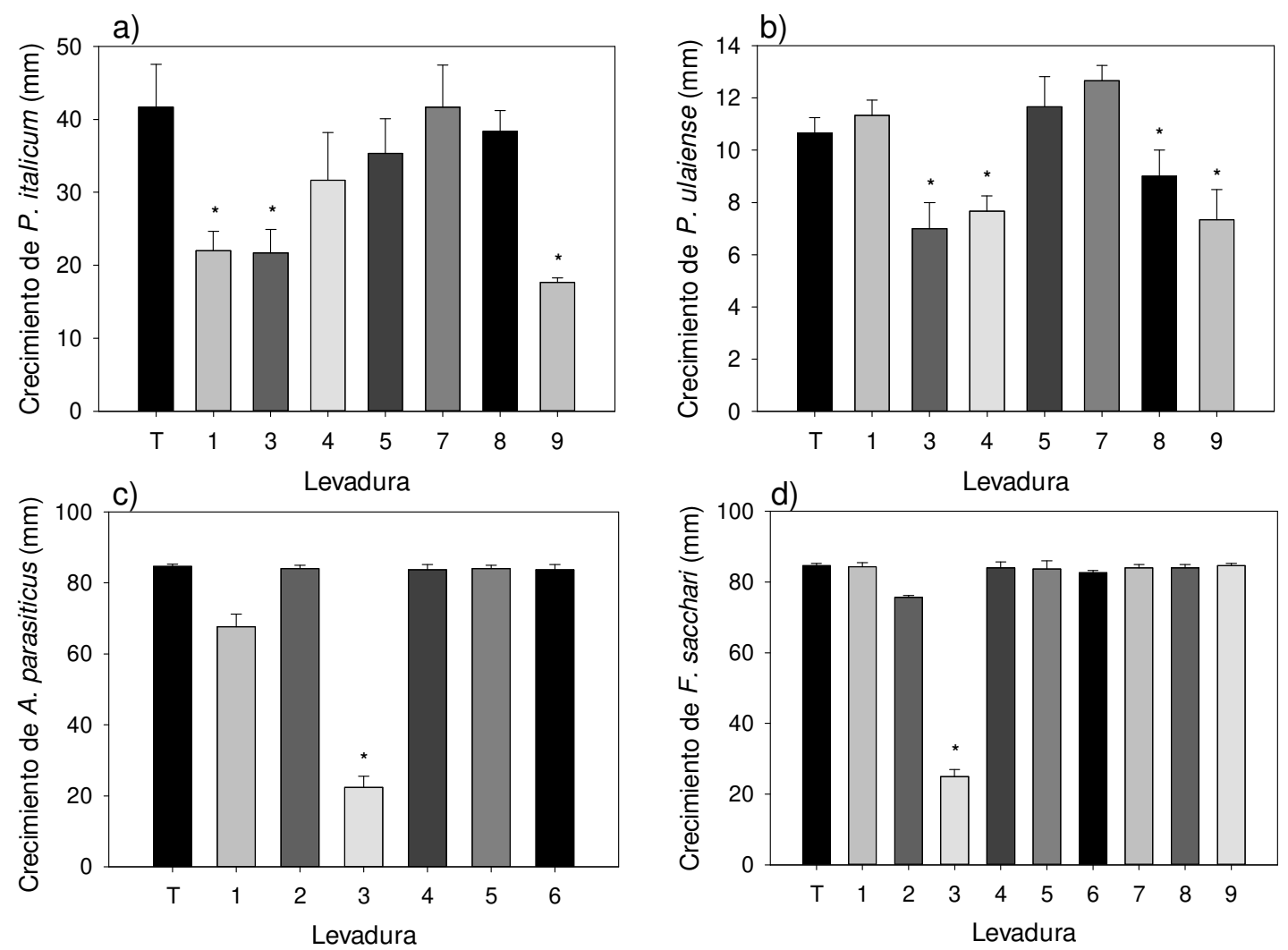

Figura 1. Diámetros de crecimiento de los mohos versus levaduras y el testigo sin inocular a los 7 días a $25^{\circ} \mathrm{C}$. a) Levaduras frente a Penicillium italicum; b) Levaduras frente a Penicillium ulaiense; c) Levaduras frente a Aspergillus parasiticus; $y$ d) Levaduras frente a Fusarium sacchari. 1 Candida parapsilosis, 2 Zygosaccharomyces bailii, 3 Lachancea thermotolerans, ${ }^{4}$ Lachancea fermentati, ${ }^{5}$ Torulaspora delbrueckii, 6 Tausonia pullulans, ${ }^{7}$ Torulaspora delbrueckii, ${ }^{8}$ Zygosaccharomyces mellis, ${ }^{9}$ Torulaspora delbrueckii $y^{T}$ testigo sin levadura. * medias significativamente diferentes a la media del testiao $(p<0,05)$. 
En cuanto a la levadura $\mathrm{N}^{\circ} 1$ identificada como $C$. parapsilosis presentó antagonismo sólo frente a Penicillium italicum (Figura 1a). La levadura $N^{\circ} 4, L$. fermentati presentó antagonismo frente a $P$. ulaiense (Figura 1b; Figura 2f) y produjo una leve disminución del crecimiento micelial a $P$. italicum (Figura 2c), aunque no manifestó diferencias con el testigo (Figura 1a).
También presentaron disminución del crecimiento del moho, la levadura $N^{\circ} 8$ : $Z$. mellis frente $P$. ulaiense (Figura $1 b$ ) y la levadura $N^{\circ} 9: T$. delbrueckii frente a $P$. italicum y $P$. ulaiense (Figura 1a y b). En la Figura 2l, se observa como $F$. sacchari, invade casi toda la placa de Petri donde se encontraba sembrada la levadura $N^{\circ} 9$, no manifestando antagonismo.
घ)

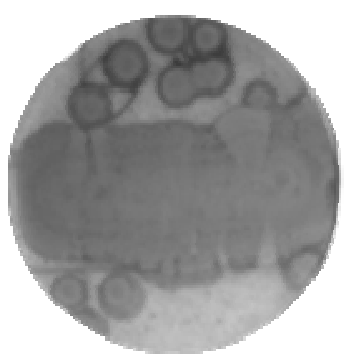

d)

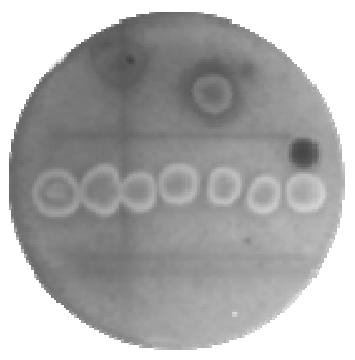

g)

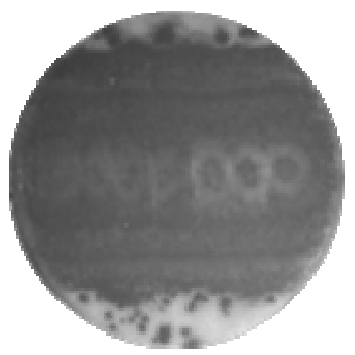

j)

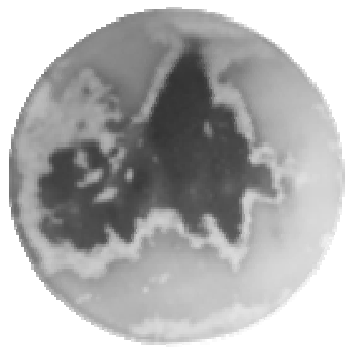

b)

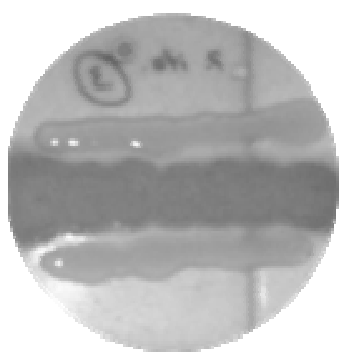

e)

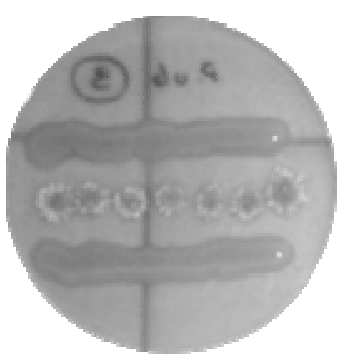

hi)

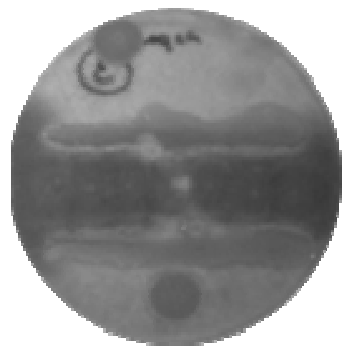

k)

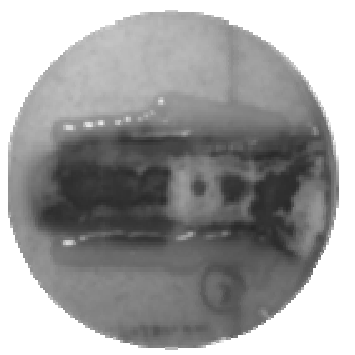

c]

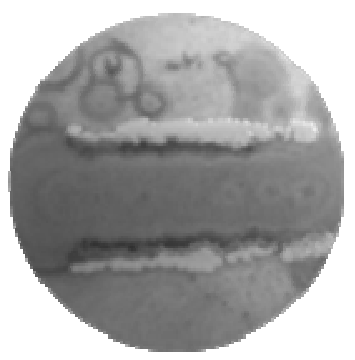

f)

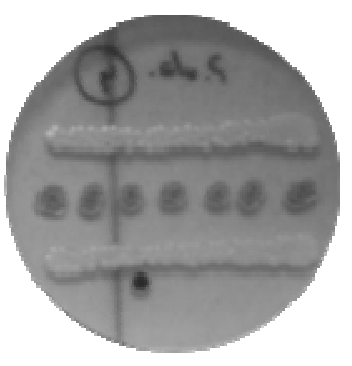

i)

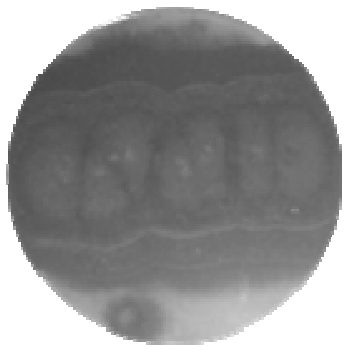

l)

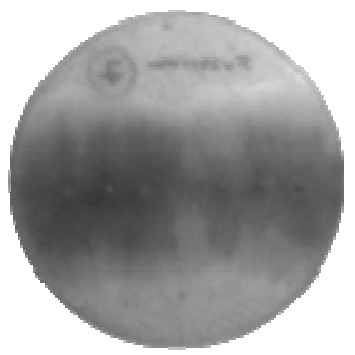

igura 2. Crecimiento de los diferentes mohos versus levaduras a los 7 días a $25^{\circ} \mathrm{C}$. a) P. italicum (testigo); b) L. Iermotolerans (levadura $N^{\circ} 3$ ) frente a $P$. Italicum; c) L. fermentati (levadura $N^{\circ} 4$ ) frente a $P$. Italicum; $d$ ) $P$. ulaiense estigo); e) L. thermotolerans (levadura $N ` 3$ ) frente a $P$. ulaiense; f) L. fermentati (levadura $\left.N{ }^{\circ} 4\right)$ frente a $P$. ulaiense; $g$ ) $A$. arasiticus (testigo); h) L. thermotolerans (levadura $N^{\circ} 3$ ) frente a $A$. parasiticus; i) Torulaspora delbrueckii (levadura $N^{\circ} 5$ ) ente a A. parasiticus; j) F. sacchari (testigo); k) L. thermotolerans (levadura No3) frente a F. sacchari; y I) Torulaspora slbrueckii (levadura $N^{\circ}$ ) frente a F. sacchari. 


\section{DISCUSIÓN}

Todas las muestras de mieles presentaron una humedad menor a 17,5\%, habiendo una baja probabilidad de fermentación en almacenamiento a una temperatura de $25^{\circ} \mathrm{C}$; siempre que la humedad se mantenga por debajo del 19-20\% (Snowdon \& Cliver, 1996; Codex Alimentarius, 2001; El Sohaimy et al., 2015). El rango de $\mathrm{pH}$ de las mieles $(3,4-5,3)$ se encontró en concordancia con el rango de $\mathrm{pH}$ de otras mieles de origen floral de la región noroeste de Argentina y otras partes del mundo (Déak, 2008; Rios et al., 2014).

La miel, generalmente, está protegida del deterioro microbiano al tener un contenido de azúcar de más del $80 \%$, pH bajo y compuestos antimicrobianos, sin embargo, es posible aislar levaduras provenientes de fuentes primarias de contaminación, como el néctar, polen y abejas, pero, también de fuentes secundarias durante la cosecha y manipuleo para su comercialización (Snowdon \& Cliver, 1996; Déak, 2009).

En este trabajo se buscó el aislamiento de levaduras provenientes de fuentes primarias de contaminación de la miel, resistentes a estrés abiótico y osmotolerantes; es decir, que no posean un requerimiento absoluto de solutos no iónicos (como la glucosa) para su viabilidad y sean capaces de tolerar un amplio rango de concentración de azúcares (Dakal et al., 2014). Los organismos osmofílicos tienen un requisito absoluto de solutos entre $10-60 \%(\mathrm{p} / \mathrm{p})$ de glucosa, por lo tanto, no crecen en medios de cultivo convencionales (Dakal et al., 2014; Čadež et al., 2015).

El agar MEA, es el medio más simple para crecimiento y enumeración de levaduras (Pitt \& Hocking, 2009) y el agar DG18 se usa particularmente en productos con una actividad agua intermedia, pues el agar czapekglicerol sólo permite el crecimiento de levaduras osmófilicas que asimilan nitratos (Déak 2008; Pitt \& Hocking, 2009). Si bien algunas especies osmotolerantes del género Zygosaccharomyces pueden desarrollarse en medios que no posean una baja actividad agua después de un periodo de adaptación (Déak, 2008; Bubnová et al., 2014), otras especies y géneros se pueden aislar directamente desde su hábitat sin la utilización de medios selectivos (Spencer \& Spencer, 1997; Sipiczki, 2003; Saksinchai et al., 2012).

Los géneros Saccharomyces, Schizosaccharomyces, Zygosaccharomyces, Candida y Torulaspora suelen ser descriptos como presentes en la miel (Raspor \& Zupan, 2006; Kurtzman \& James, 2006; Pitt \& Hocking, 2009). Z. mellis, es una especie que puede llegar a fermentar concentraciones de azúcar como las normalmente presentes en la miel, donde otras levaduras se verían inhibidas por efecto de la alta presión osmótica (Liu et al., 2016).

T. pullulans, es repetidamente identificada en suelo, hojas y frutos, como una especie dominante (Zhang et al., 2017; Wang et al., 2018). Los géneros descriptos presentes en miel en general son encontrados en el polen, néctar, suelo, polvo y flores; ya que son dispersados con la ayuda de agentes como el viento y los insectos, en este caso abejas (Olaitan et al., 2007).
Se procedió a probar el antagonismo de algunas de las levaduras aisladas, contra mohos descriptos como patógenos poscosecha de cítricos $(P$. italicum y $P$. ulaiense) y otros patógenos precosecha de maíz, maní y caña de azúcar ( $A$. parasiticus y $F$. sacchari). Estos géneros de mohos, también, son los más comúnmente encontrados en miel de abejas y polen (González et al., 2005; Kačániová et al., 2012; Salgado Silva et al., 2017).

Se ensayó el antagonismo de levaduras de miel frente a mohos patógenos, porque suelen emplearse levaduras de distinto origen (flores, frutos, filoplano, suelo, raíces, insectos, agua de mar), en el control biológico de los de los mismos (Liu et al., 2013a; Liu et al., 2013b; Kurtzman \& Sugiyama, 2015; Perez et al., 2016). Además, al tratarse de levaduras osmotolerantes, generalmente inocuas y con capacidad de mantenerse viables en las condiciones antimicrobianas de la miel (Snowdon \& Cliver, 1996; Aurongzeb \& Azim, 2011; Vázquez-Quiñones et al., 2018) y del néctar de las plantas, en presencia de fitoquímicos y alta presión osmótica (Herrera et al., 2009; Herrera et al., 2010), serían buenas candidatas para ser utilizadas en el control de mohos patógenos, en ensayos a campo, o durante el almacenamiento de granos y semillas.

La levadura $N^{\circ} 3$ L. thermotolerans, resultó efectiva para el control in vitro de todos los mohos testeados. Esta especie, también fue aislada de la microbiota epífita de plantas de vid, siendo antagonista de $A$. carbonarius y A. niger, responsables de la acumulación de micotoxinas en uvas (Bleve et al., 2006). La levadura $N^{\circ} 1, C$. parapsilosis limitó el crecimiento sólo de $P$. italicum pero no de los demás hongos. En trabajos previos, en la Provincia de Jujuy, se encontró que especies del mismo género ( $C$. versatilis y $C$. cantarellii) aisladas de cítricos, resultaron entre las más activas in vitro, frente a $P$. digitatum (Benítez Ahrendts \& Carrillo, 2004): También, en otro trabajo en el noroeste argentino (Provincia de Tucumán) se encontró antagonismo mayor o igual al $40 \%$, en reducción del diámetro de crecimiento micelial de Penicillium italicum y $P$. citri, por acción de $C$. catenulata, y otras especies aisladas de frutos y hojas de cítricos (Perez et al., 2016).

Asimismo, cepas de C. catenulata, C. famata, C. sake, $C$. versatillis, $K$. marxianus y $T$. delbrueckii, presentaron algún tipo de antagonismo frente a los mohos de la podredumbre de las uvas, Rhizopus stolonifer, $A$. terreus, $A$. versicolor, $A$. caelatus y $P$. commune, mediante inhibición del crecimiento micelial, exclusión competitiva, producción de enzimas líticas de pared, inhibición de la germinación de esporas y producción de sideróforos (Nally et al., 2015). Por lo que serían necesarios otros ensayos in vitro, para poder determinar la naturaleza del antagonismo de las levaduras aisladas, hacia los mohos.

La levadura $N^{\circ} 4, L$. fermentati presentó antagonismo frente a $P$. ulaiense (Figura 1b), y disminución del crecimiento micelial frente a $P$. italicum (Figura 2c), aunque no suficiente para detectarse diferencias con el testigo $(p<0,05)$, por la producción de algún metabolito (Castoria et al., 1997). 
También presentó disminución del crecimiento del moho, la levadura $N^{\circ} 8$ Z. mellis, aislada de miel de abeja melipona, frente $P$. ulaiense (Figura 1b). Este género de levaduras se encuentra descripto en mieles y abejas meliponas sanas; y junto a otras especies de levaduras, actuarían en relaciones mutualistas con las mismas, por ejemplo, deshidratar los granos de polen, para retardar su deterioro (Ganter, 2006; Morais et al., 2013).

Se encontró que las levaduras $N^{\circ} 5,7$ y 9, identificadas como $T$. delbrueckii, al ensayarse su actividad frente a $P$. italicum y $P$. ulaiense, sólo la levadura $N^{\circ} 9$ manifestó actividad hacia $P$. italicum; en las mismas condiciones de experimentación. Este hallazgo, estuvo en concordancia con Suzzi et al. (1995), donde se observa que la actividad de biocontrol de levaduras del vino, contra mohos patógenos, es una característica relacionada con la cepa y no depende únicamente de la especie o género de la levadura. Nally et al. (2015) también observó un comportamiento diferencial de cepas como antagonistas de mohos aislados de uvas en descomposición, por ejemplo, de tres cepas de $T$. delbrueckii; dos presentaron exclusión competitiva por producción de metabolitos frente a Penicillium commune.

Si bien se utilizó miel para el aislamiento de bacterias productoras de antibióticos con actividad hacia patógenos de abejas (Sabaté et al., 2009), este es el primer trabajo donde se utilizó esta aproximación para la selección de levaduras antagónicas de mohos patógenos pre- y poscosecha de diversos cultivos, como cítricos, maíz, maní y caña de azúcar.

Los resultados de este estudio, indican que en la microbiota asociada a mieles de la provincia de Jujuy hay levaduras antagonistas de mohos patógenos pre- y poscosecha, posiblemente a través del mecanismo de exclusión competitiva de nutrientes o por producción de algún metabolito o enzima lítica de pared fúngica; por lo que son necesarias otras pruebas in vitro para elucidar la naturaleza del antagonismo.

\section{CONCLUSIÓN}

Se logró aislar un total de 15 levaduras de 25 muestras de mieles de la provincia de Jujuy. Todas pertenecieron a géneros normalmente presentes en mieles, o ambiente circundante. De los aislamientos obtenidos, cinco presentaron antagonismo hacia mohos descriptos como patógeno pre- y poscosecha de cultivos.

El antagonismo se observó por disminución del crecimiento micelial de los mohos. Las levaduras que tuvieron acción antagónica fueron: Lachancea thermotolerans frente a Aspergillus parasiticus, Fusarium sacchari, Penicillium italicum y $P$. ulaiense. Candida parapsilosis frente a $P$. italicum, Lachancea fermentati frente a $P$. ulaiense, $Z$. mellis frente a $P$. ulaiense, y $T$. delbrueckii frente a $P$. italicum y $P$. ulaiense.

Se podrían utilizar las levaduras antagonistas, en futuros ensayos in vivo, en vistas al diseño de un biofungicida activo contra mohos patógenos de cultivos, en la etapa de producción a campo, o durante el almacenamiento.

\section{AGRADECIMIENTOS}

FCA-UNJu y SeCTER-UNJu.

\section{BIBLIOGRAFÍA}

Al-Qaysi, S. A., H. Al-Haideri, Z. A. Thabit, W. H. A. A. Al-Kubaisy \& J. A. A. R. Ibrahim. 2017. Production, Characterization, and Antimicrobial Activity of Mycocin Produced by Debaryomyces hansenii DSMZ70238. International Journal of Microbiology 2017: 1-9.

Aurongzeb, M. \& M. K. Azim. 2011. Antimicrobial properties of natural honey: a review of literature. Pakistan Journal of Biochemistry \& Molecular Biology 44 (3): 118-124.

Bajaj, B. K., S. Raina \& S. Singh. 2012. Killer toxin from a novel killer yeast Pichia kudriavzevii RY55 with idiosyncratic antibacterial activity. Journal of Basic Microbiology 53 (8): 645-656.

Bakier S. 2009. Capabilities of near-infrared spectroscopy to analyze changes in water bonding during honey crystallization process. International Journal of Food Science \& Technology 44: 519-524.

Benítez Ahrendts, M. R. \& L. Carrillo. 2004. Levaduras inhibitorias de Penicillium. Revista Argentina de Microbiología 36: 182-186.

Bleve, G., F. Grieco, G. Cozzi, A. Logrieco \& A. Visconti. 2006. Isolation of epiphytic yeasts with potential for biocontrol of Aspergillus carbonarius and $A$. niger on grape. International Journal of Food Mcrobiology 108 (2): 204-209.

Bubnová, M., J. Zemančíková \& H. Sychrová. 2014. Osmotolerant yeast species differ in basic physiological parameters and in tolerance of non-osmotic stresses. Yeast 31 (8): 309-321.

Čadež, N., L. Fülöp, D. Dlauchy \& G. Péter. 2015. Zygosaccharomyces favi sp. nov., an obligate osmophilic yeast species from bee bread and honey. Antonie Van Leeuwenhoek 107 (3): 645-654.

Canadian Food Inspection Agency. 2019. Operational procedure: Honey inspection procedures. Appendix 2: Honey conversion table: ${ }^{\circ}$ Brix, specific gravity and \% moisture relationships [Online]. Disponible en: http://www.inspection.gc.ca/food/compliancecontinuum/guidance-for-inspectors/sip/honeyinspectionprocedures/eng/1539272153253/1539272153460\#a72. Último acceso: mayo de 2019.

Castoria, R., F. De Curtis, G. Lima \& V. De Cicco. 1997. $\beta-1$, 3-glucanase activity of two saprophytic yeasts and possible mode of action as biocontrol agents against postharvest diseases. Postharvest Biology and technology 12 (3): 293-300.

Castoria, R., F. De Curtis, G. Lima, L. Caputo, S. Pacifico \& V. De Cicco. 2001. Aureobasidium pullulans (LS-30) an antagonist of postharvest pathogens of fruits: study on its modes of action. Postharvest Biology and Technology 22 (1): 7-17.

Chen, W. J., F. Delmotte, S. Richard-Cervera, L. Douence, C. Greif \& M. F. Corio-Costet. 2007. At least two origins of fungicide resistance in grapevine downy 
Mildew populations. Applied Environmental Microbiology 73 (16): 5162-5172.

Codex Alimentarius Commission. 2001. Codex Standard for Honey (Adopted in 1981, Revisions 1987 and 2001). Food and Agriculture Organization of the United Nations and World Health Organization. Codex Stan 12 (1981): 1-8.

Dakal, T. C., L. Solieri \& P. Giudici. 2014. Adaptive response and tolerance to sugar and salt stress in the food yeast Zygosaccharomyces rouxii. International Journal of Food Microbiology 185: 140-157.

Déak, T. 2008. Yeasts in Specific Types of Foods. Handbook of Food Spoilage Yeasts. 2nd Ed. CRC Press, Boca Raton. pp. 117-201.

Döll, S. \& S. Dänicke. 2011. The Fusarium toxins deoxynivalenol (DON) and zearalenone (ZON) in animal feeding. Preventive veterinary medicine 102 (2): 132145.

Droby, S., M. Wisniewski, D. Macarisin \& C. Wilson. 2009. Twenty years of postharvest biocontrol research: is it time for a new paradigm?. Postharvest Biology and Technology 52 (2): 137-145.

El Sohaimy, S. A., S. H. D. Masry \& M. G. Shehata. 2015. Physicochemical characteristics of honey from different origins. Annals of Agricultural Sciences 60 (2): 279-287.

Ganter, P. F. 2006. Yeast and invertebrate associations. En: Biodiversity and ecophysiology of yeasts. Rosa, C. \& G. Péter, Eds. Springer-Verlag, Berlín pp. 303-370.

Giobbe, S., S. Marceddu, B. Scherm, G. Zara, V. L. Mazzarello, M. Budroni \& Q. Migheli. 2007. The strange case of a biofilm-forming strain of Pichia fermentans, which controls Monilinia brown rot on apple but is pathogenic on peach fruit. FEMS Yeast Research 7 (8): 1389-1398.

Gomes, T., X. Feás, A. Iglesias \& L. M. Estevinho. 2011. Study of organic honey from the northeast of Portugal. Molecules 16 (7): 5374-5386.

González, G., M. J. Hinojo, R. Mateo, A. Medina \& M. Jiménez. 2005. Occurrence of mycotoxin producing fungi in bee pollen. International Journal of Food Microbiology 105 (1): 1-9.

Guo, J., W. Fang, H. Lu, R. Zhu, L. Lu, X. Zheng \& T. $\mathbf{Y u}$. 2014. Inhibition of greenmold disease in mandarins by preventive applications of methyl jasmonate and antagonistic yeast Cryptococcus laurentii. Postharvest Biology and Technology 88: 72-78.

Herrera, C. M., C. de Vega, A. Canto \& M. I. Pozo. 2009. Yeasts in floral nectar: a quantitative survey. Annals of Botany 103 (9): 1415-1423.

Herrera, C. M., A. Canto, M. I. Pozo \& P. Bazaga. 2010. Inhospitable sweetness: nectar filtering of pollinator-borne inocula leads to impoverished, phylogenetically clustered yeast communities. Proceedings of the Royal Society B: Biological Sciences 277 (1682): 747-754.

Hu, H., M. E. Wisniewsk, A. Abdelfattah \& X. Zheng. 2017. Biocontrol activity of a cold-adapted yeast from Tibet against gray mold in cherry tomato and its action mechanism. Extremophiles 21(4): 789-803.

Hua, S. S. T., J. L. Baker \& M. Flores Espíritu. 1999. Interactions of saprophytic yeast with a nor mutant of Aspergillus flavus. Applied and Environmental Microbiology 65: 2738-2740.
Huang, R., G. O. Li, J. Zhang, L. Yang, H. J. Che, D. H. Jiang \& H. C. Huang. 2011. Control of postharvest Botrytis fruit rot of strawberry by volatile organic compounds of Candida intermedia. Phytopathology 101: 859-869.

Janisiewicz, W. J., T. J. Tworkoski \& C. Sharer. 2000. Characterizing the mechanism of biological control of postharvest diseases on fruits with a simple method to study competition for nutrients. Phytopathology 90 (11): 1196-1200.

Kačániová, M., V. Kňazovická, S. Felšöciová \& K. Rovná. 2012. Microscopic fungi recovered from honey and their toxinogenity. Journal of Environmental Science and Health, Part A 47 (11): 1659-1664.

Kurtzman, C., J. W. Fell \& T. Boekhout. 2011. The Yeasts: a taxonomic study. 5th Ed. Elsevier, London. 2354 pp.

Kurtzman, C. P. \& S. A. James. 2006. Zygosaccharomyces and related genera. En: Food spoilage microorganisms. Blackburn C. de W., Ed. Woodhead Publishing, Cambridge pp. 289-305.

Kurtzman, C. P. \& J. Sugiyama. 2015. Saccharomycotina and Taphrinomycotina: the yeasts and yeastlike fungi of the Ascomycota. En: The Mycota. VII Systematics and Evolution. McLaughlin, D. J. \& J. W. Spatafora, Eds. 2da. Ed. Springer-Verlag, BerlinHeidelberg pp. 3-33.

Leslie, J. F. \& B. A. Summerell. 2006. Fusarium laboratory workshops - A recent history. Mycotoxin Research 22 (2): 73-74.

Liu, J., Y. Sui, M. Wisniewski, S. Droby \& Y. Liu. 2013a. Utilization of antagonistic yeasts to manage postharvest fungal diseases of fruit. International Journal of Food Microbiology 167 (2): 153-160.

Liu, G. L., Z. Chi, G. Y. Wang, Z. P. Wang, Y. Li \& Z. M. Chi. 2013b. Yeast killer toxins, molecular mechanisms of their action and their applications. Critical Reviews in Biotechnology 35 (2): 222-234.

Liu, G., C. Tao, B. Zhu, W. Bai, L. Zhang, Z. Wang \& X. Liang. 2016. Identification of Zygosaccharomyces mellis strains in stored honey and their stress tolerance. Food science and biotechnology 25 (6): $1645-1650$.

Lutz, M. C., C. A. Lopes, M. E. Rodriguez, M. C. Sosa \& M. P. Sangorrín. 2013. Efficacy and putative mode of action of native and commercial antagonistic yeasts against postharvest pathogens of pear. International Journal of Food Microbiology 164 (2-3): 166-172.

Mandal, M. D. \& S. Mandal. 2011. Honey: its medicinal property and antibacterial activity. Asian Pacific Journal of Tropical Biomedicine 1 (2): 154-160.

Marquina, D., A. Santos \& J. Peinado. 2002. Biology of killer yeasts. International Microbiology 5 (2): 65-71.

Martins, L. M., A. S. Sant'Ana, M. H. Pelegrinelli Fungaro, J. J. Silva, M. da Silva do Nascimento, J. C. Frisvad \& M. H. Taniwaki. 2017. The biodiversity of Aspergillus section Flavi and aflatoxins in the Brazilian peanut production chain. Food Research International 94: 101-107.

Morais, P. B., P. S. S. T. Calaça \& C. A. Rosa. 2013. Microorganisms associated with stingless bees. En: Pot-Honey. Vit, P. \& S. R. M. Pedro, Eds. Springer, New York pp. 173-186.

Moretti, A., G. Mule, A. Ritieni \& A. Logrieco. 2007. Further data on the production of beauvericin, enniatins 
and fusaproliferin and toxicity to Artemia salina by Fusarium species of Gibberella fujikuroi species complex. International Journal of Food Microbiology 118 (2): 158-163.

Nally, M. C., V. M. Pesce, Y. P. Maturano, C. J. Muñoz, M. Combina, M. E. Toro, L. C. Castellanos de Figueroa \& F. Vazquez. 2012. Biocontrol of Botrytis cinerea in table grapes by non-pathogenic indigenous Saccharomyces cerevisiae yeasts isolated from viticultural environments in Argentina. Postharvest biology and technology 64 (1): 40-48.

Nally, M. C., V. M. Pesce, Y. P. Maturano, L. R. Assaf, M. E. Toro, L. C. Castellanos de Figueroa \& F. Vazquez. 2015. Antifungal modes of action of Saccharomyces and other biocontrol yeasts against fungi isolated from sour and grey rots. International Journal of Food Microbiology 204: 91-100.

Nunes, C. A. 2012. Biological control of postharvest diseases of fruit. European Journal of Plant Pathology 133: 181-196.

Olaitan, P. B., O. E. Adeleke \& O. O. Iyabo. 2007. Honey: a reservoir for microorganisms and an inhibitory agent for microbes. African Health Sciences 7 (3): 159165.

Palou, L., J. L. Smilanick \& S. Droby. 2008. Alternatives to conventional fungicides for the control of citrus postharvest green and blue moulds. Stewart Postharvest Review 4 (2): 1-16.

Perez, M. F., L. Contreras, N. M. Garnica, M. V. Fernández-Zenoff, M. E. Farías, M. Sepulveda, J. Ramallo \& J. R. Dib. 2016. Native killer yeasts as biocontrol agents of postharvest fungal diseases in lemons. PLoS One 11 (10): e0165590.

Pitt, J. I. \& A. Hocking. 2009. Fungi and Food Spoilage. 3rd Ed. Springer, Boston 519 pp.

Platania, C., C. Restuccia, S. Muccilli \& G. Cirvilleri. 2012. Efficacy of killer yeasts in the biological control of Penicillium digitatum on Tarocco orange fruits (Citrus sinensis). Food microbiology 30 (1): 219-225.

Ploetz, R. C. 2006. Fusarium-induced diseases of tropical, perennial crops. Phytopathology 96 (6): 648652.

Raspor, P. \& J. Zupan. 2006. Yeasts in Extreme Environments. En: Biodiversity and ecophysiology of yeasts. Rosa, C. \& G. Péter, Eds. Springer-Verlag, Berlín pp. 371-417.

Rios, F., A. C. Sanchez, M. Lobo, L. Lupo, I. Coelho, I. Castanheira \& N. Samman. 2014. A chemometric approach: characterization of quality and authenticity of artisanal honeys from Argentina. Journal of Chemometrics 28 (12): 834-843.

Robert, V., G. Stegehuis \& J. Stalpers. 2004. The MycoBank engine and related databases [Online]. Disponible en: http://www.mycobank.org. Último acceso: mayo de 2019.

Sabaté, D. C., L. Carrillo \& C. M. Audisio. 2009. Inhibition of Paenibacillus larvae and Ascosphaera apis by Bacillus subtilis isolated from honeybee gut and honey samples. Research in Microbiology 160 (3): 193199.

Saksinchai, S., M. Suzuki, P. Chantawannakul, M. Ohkuma \& S. Lumyong. 2012. A novel ascosporogenous yeast species, Zygosaccharomyces siamensis, and the sugar tolerant yeasts associated with raw honey collected in Thailand. Fungal Diversity 52 (1): 123-139.

Salgado Silva, M., Y. Rabadzhiev, M. R. Eller, I. Iliev, I. Ivanova \& W. C. Santana. 2017. Microorganisms in honey. Honey Analysis. Toledo V. A. A., Ed. IntechOpen, DOI: 10.5772/67262. Disponible en: https://www.intechopen.com/books/honey-

analysis/microorganisms-in-honey. Último acceso: junio de 2019.

Sánchez-Torres, P. \& J. J. Tuset. 2011. Molecular insights into fungicide resistance in sensitive and resistant Penicillium digitatum strains infecting citrus. Postharvest Biology Technology 59 (2): 159-165.

Sharma, R. R., D. Singh \& R. Singh. 2009. Biological control of postharvest diseases of fruits and vegetables by microbial antagonists: A review. Biological control 50 (3): 205-221.

Snowdon, J. A. \& D. O. Cliver. 1996. Microorganisms in honey. International Journal of Food Microbiology 31: 1-26.

Spencer J. F. T. \& D. M. Spencer. 1997. Ecology: Where Yeasts Live. En: Yeasts in Natural and Artificial Habitats. Spencer, J. F. T. \& D. M. Spencer, Ed.. Springer-Verlag, Berlin pp. 33-58.

Sipiczki, M. 2003. Candida zemplinina sp. nov., an osmotolerant and psychrotolerant yeast that ferments sweet botrytized wines. International Journal of Systematic and Evolutionary Microbiology 53 (6): 20792083.

Suzzi, G., P. Romano, L. Ponti \& C. Montuschi. 1995. Natural wine yeasts as biocontrol agents. Journal of Applied Bacteriology 78: 304-308.

Tripathi, P. \& N. Dubey. 2003. Exploitation of natural products as an alternative strategy to control postharvest fungal rotting of fruit and vegetables. Postharvest Biology Technology 32 (3): 235-245.

Vázquez-Quiñones, C. R., R. Moreno-Terrazas, I. Natividad-Bonifacio, E. I. Quiñones-Ramírez \& C. Vázquez-Salinas. 2018. Microbiological assessment of honey in México. Revista Argentina de Microbiologia 50 (1): 75-80.

Velásquez Giraldo, A. M., L. M. Vélez Acosta \& R. Zuluaga Gallego. 2013. Physicochemical and microbiological characterization of Apis mellifera sp. honey from southwest of Antioquia in Colombia. Ingeniería y Ciencia 9 (18): 61-74.

Vica, M., M. Glevitzky, G. A. Dumitrel, M. Popa \& S. Varvara. 2009. Microbiological role in hazards analysis of natural honey processing. Journal of Agroalimentary Processes and Technologies 15 (3):353-360.

Vallianou, N. G., P. Gounari, A. Skourtis, J. Panagos \& C. Kazazis. 2014. Honey and its anti-inflammatory, anti-bacterial and anti-oxidant properties. General Medicine (Los Angeles) 2 (132): 1-5.

Viswanathan, R., M. Poongothai \& P. Malathi. 2011. Pathogenic and molecular confirmation of Fusarium sacchari causing wilt in sugarcane. Sugar Tech 13 (1): 68-76.

Wang, Z., J. Zhang, F. Wu \& X. Zhou. 2018. Changes in rhizosphere microbial communities in potted cucumber seedlings treated with syringic acid. PloS one 13 (6): e0200007.

Westerdijk Fungal Biodiversity Institute. 2019. Polyphasic identification [Online]. Disponible en: 
Revista de la Facultad de Agronomía, La Plata (2020) Vol 119 (2): 1-11

http://www.westerdijkinstitute.nl/Collections/BiolomicsID. aspx?IdentScenario=Yeast2011ID. Último acceso: mayo de 2019.

Williams, J. H., T. D. Phillips, P. E. Jolly, J. K. Stiles, C. M. Jolly \& D. Aggarwal. 2004. Human aflatoxicosis in developing countries: a review of toxicology, exposure, potential health consequences, and interventions. The American journal of clinical nutrition 80 (5): 1106-1122.
Yao, H. J. \& S. P. Tian. 2005. Effects of a biocontrol agent and methyl jasmonate on postharvest diseases of peach fruit and the possible mechanisms involved. Journal of Applied Microbiology 98 (4): 941-950.

Zhang, S., X. Chen, Q. Zhong, Z. Huang \& Z. Bai. 2017. Relations among epiphytic microbial communities rom soil, leaves and grapes of the grapevine. Frontiers in Life Science 10 (1): 73-83. 